\title{
Influence of Ag content on mechanical and tribological behavior of DLC coatings
}

\author{
N.K. Manninen a,*, F. Ribeiro a, A. Escudeiro a, T. Polcar ${ }^{\text {b,c }}$, S. Carvalho ${ }^{\mathrm{a}, \mathrm{d}}$, A. Cavaleiro ${ }^{\mathrm{a}}$ \\ a SEG-CEMUC, Mechanical Engineering Department, University of Coimbra, 3030-788 Coimbra, Portugal \\ ${ }^{\mathrm{b}}$ National Centre for Advanced Tribology at Southampton (nCATS), School of Engineering Sciences, University of Southampton, Highfield, Southampton SO17 1BJ, Hampshire, UK \\ c Department of Control Engineering, Faculty of Electrical Engineering, Czech Technical University in Prague, Technická 2, Prague 6, Czech Republic \\ d GRF-CFUM, Universidade do Minho, Dept. Física, Campus de Azurém, 4800-058 Guimarães, Portugal
}

\section{A R T I C L E I N F O}

\section{Article history:}

Received 6 February 2013

Accepted in revised form 28 May 2013

Available online 11 June 2013

\section{Keywords:}

Ag-DLC

Structure

Mechanical properties

Tribology

Wear mechanisms

\begin{abstract}
A B S T R A C T
Ag-DLC coatings with Ag contents ranging from 1.3 at.\% to 13.1 at.\% were deposited by DC magnetron sputtering. The coatings were characterized with respect to their structure (by means of XRD and Raman spectroscopy), mechanical and tribological properties (by scratch test, nanoindentation, residual stress measurements and pin-on-disk test). The incorporation of $13.1 \mathrm{at} . \% \mathrm{Ag}$ resulted in the formation of $\mathrm{Ag}$ grains of 2-3 nm which promoted the increase of graphite like bonds organized in rings. Regarding the mechanical properties, no variations were found for films with Ag contents lower than 13 at.\%; a reduction of both hardness and compressive residual stress was then observed for higher values. Pin-on-disk tests were performed at two different contact pressures (690 MPa and $1180 \mathrm{MPa}$ ) in dry sliding conditions against a zirconia counterpart. For the lower contact pressure the variations in the wear rate are well correlated with the coating structure and mechanical properties, while for the higher contact pressure the presence of Ag is relevant and Ag-DLC coatings are showing higher wear rate than DLC one. SEM analysis revealed the formation of $\mathrm{Ag}$ aggregates on the wear track and adhesion of silver to the counterpart.
\end{abstract}

(c) 2013 Elsevier B.V. All rights reserved.

\section{Introduction}

Diamond like carbon (DLC) coatings often combine high wear resistance with low friction coefficient, which has prompted their use in several industrial applications as wear protective coatings [1-3]. Moreover, these coatings are biocompatible and chemically inert and thus interesting for biomedical applications, namely hip joints [4], knee replacements [5], coronary artery stents [6] or heart valves [7]. DLC coatings consist on hydrogenated and non-hydrogenated amorphous carbons (a-C:H and a-C, respectively) with different structures according to their chemical composition. The various forms of amorphous $\mathrm{C}-\mathrm{H}$ alloys can be displayed in a ternary phase diagram and classified according to the amount of $\mathrm{sp}^{2} / \mathrm{sp}^{3}$ bonds and $\mathrm{H}$ content [8]. a-C coatings present higher hardness, better wear resistance and lower friction in humid environments in relation to a-C:H coatings [9].

Despite their excellent tribological properties, high residual stresses and consequent adhesion failure are pointed as one of the major limitations of both a-C and a-C:H coatings [10]. The incorporation of metal atoms such as Ti [11], W [12], $\mathrm{Cr}$ [13], Zr [14], Cu [2] or Ag [15-17], to carbon films could reduce residual stress and thus the risk of premature

\footnotetext{
* Corresponding author. Tel.: + 351239 790745, +351 239 790700; fax: + 351239 790701.
}

E-mail address: nora.sousa@dem.uc.pt (N.K. Manninen). coating adhesion failure. These metals might form small nanocrystallites of pure metal or metal carbide nanograins dispersed through the carbon structure [10]. The incorporation of carbide forming metals (e.g. Ti, W, $\mathrm{Cr}$, and $\mathrm{Zr}$ ) enhances the coating's adhesive strength and hardness. Alloying of DLC with noble metals (such as $\mathrm{Ag}$ and $\mathrm{Cu}$ ) results in the formation of soft and ductile phases able to improve the coating's adhesion and toughness [2,16]. In fact, DLC coatings alloyed with Ag exhibited enhanced coating adhesion to steel substrates [16] and reduced internal stresses [17]. Moreover, these soft metals could act as solid lubricants further decreasing the friction coefficient.

Several works have proposed doping or alloying of hard-wear resistant coatings (e.g. TiCN [18]; CrN; TiN; ZrN [19]; DLC [15]) with Ag in order to improve the tribological behavior of these coatings. X. Yu et al. [15] found that the incorporation of 4.3 to 10.6 at.\% $\mathrm{Ag}$ into DLC coatings resulted in a decrease of both wear rate and friction coefficient, which was attributed to the presence of $\mathrm{Ag}$ nanograins on the top layer of the Ag-DLC coating. Higher Ag contents lead to unsatisfactory tribological properties with high friction and low wear resistance. In addition to the promising tribological properties, $\mathrm{Ag}$ is a potential antibacterial agent often studied and used in the biomedical field $[16,17]$. The combination of wear resistant coatings ( $\mathrm{CrN}$, TiN, ZrN [19]; TiCN [18]; TaN [20]; DLC [21]) with silver has been proposed as an effective solution able to improve the resistance to bacterial colonization and, simultaneously, the wear resistance of the base coatings. 
In the present study, the effect of $\mathrm{Ag}$ content on the mechanical and tribological properties of Ag-DLC coatings is evaluated. Ag-DLC coatings were deposited by magnetron sputtering and the influence of chemical composition on coatings' structure, mechanical and tribological properties was evaluated. Pin-on-disk tests were performed in dry sliding conditions against a zirconia counterpart at two different contact pressures.

\section{Experimental procedure}

Ag-DLC coatings were deposited by DC magnetron sputtering from a graphite target $\left(200 \times 100 \mathrm{~mm}^{2}\right)$ onto polished and ultrasonically cleaned tool steel (used for tribological tests), stainless steel (determination of residual stress and XRD analysis), high-speed steel (AISI M2) (for determination of coating adhesion) and silicon (nanoindentation tests and Raman spectroscopy). High purity silver pellets (with an average area of approximately $20 \mathrm{~mm}^{2}$ ) were incorporated in the erosion zone of the graphite target. In order to vary the silver content different numbers of silver pellets from 1 to 11 were used, as can be depicted in Table 1 . The depositions were carried out in an $\mathrm{Ar}$ atmosphere with substrates rotating at $17 \mathrm{~cm}$ from the target at a constant speed of $18 \mathrm{rpm}$. The base pressure in the deposition chamber was approximately $9 \times 10^{-4} \mathrm{~Pa}$ and rose up to values of approximately $5 \times 10^{-1} \mathrm{~Pa}$ during the deposition. The target power density was kept at $7.5 \mathrm{~W} / \mathrm{cm}^{2}$ (considering the total target area). The coatings were deposited at room temperature with a target bias voltage of $-50 \mathrm{~V}$, the argon flow was $45 \mathrm{sccm}$. Prior to Ag-DLC deposition a Ti/TiN/TiCN gradient interlayer with a thickness of $450 \mathrm{~nm}$ was previously deposited in order to improve the coating adhesion. For more details see Ref. [14].

The chemical composition of the deposited films was investigated by a Cameca SX 50 electron probe microanalysis (EPMA) apparatus. Ball crater tests were used to measure the film thickness. The silver structure and grain size were determined by X-ray diffraction (XRD) using a conventional Philips X'Pert diffractometer, operating with CoK $\alpha$ radiation in grazing incidence mode with an angle of $2^{\circ}$. To analyze the chemical bonding of DLC matrix, Raman spectra were acquired using Horiba Yvon Xplora with an excitation light of $532 \mathrm{~nm}$ (diode pumped solid state (DPSS) laser) and a laser power of $2 \mathrm{~mW}$; the integration time was $90 \mathrm{~s}$ with 3 accumulations. The coating adhesion was evaluated by, scratch test performed with a diamond Rockwell indenter; the load linearly increased in the range 2-50 N applied with a rate of $10 \mathrm{~N} / \mathrm{mm}$. Residual stresses were determined from Stoney's equation [22], using the substrate curvature radii before and after the coating deposition. Coating's hardness was evaluated by MicroMaterials Nanotest system equipped with a Berkovich indenter with a maximum load of $5 \mathrm{mN}$, which resulted in an indentation depth of approximately $10 \%$ of the film thickness. The hardness value was an average of 48 points measured. The tribological properties of Ag-DLC coatings were studied using a pin-on-disk CSM Tribometer. The tribological tests were performed in dry sliding at room temperature and relative air humidity of $40 \%$. The tests were performed against zirconia balls, and two different mean Hertzian contact pressures $(\mathrm{Pm})$ were used: $690 \mathrm{MPa}$ (applied load of $10 \mathrm{~N}$ and a ball with a

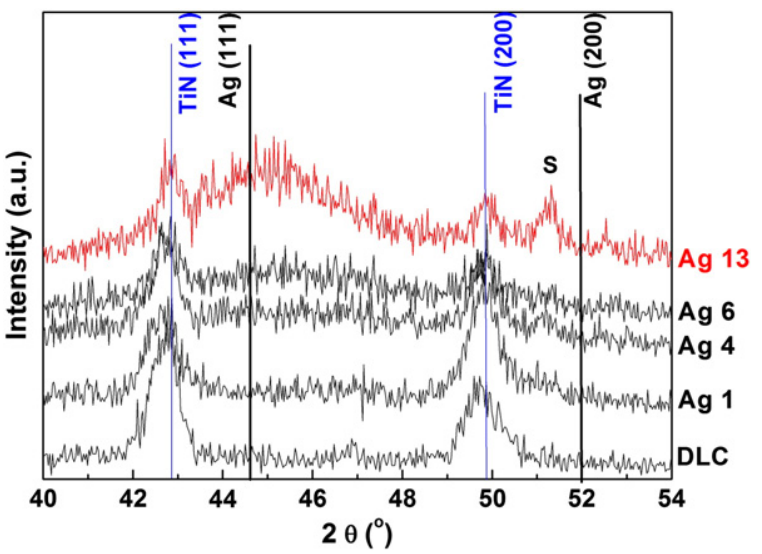

Fig. 1. XRD patterns of Ag-DLC coatings with different silver contents.

diameter of $10 \mathrm{~mm}$ ) and $1180 \mathrm{MPa}$ (applied load of $18 \mathrm{~N}$ and a ball of $6 \mathrm{~mm}$ ). For each contact pressure two tests were performed with a total of 20000 laps and a linear speed of $10 \mathrm{~cm} \cdot \mathrm{s}^{-1}$. The coating wear rate was evaluated as worn volume measured by 3D white light profilometer, per load per sliding distance [23]. The worn surfaces were analyzed by scanning electron microscopy with energy dispersive spectrometry (SEM/EDS).

\section{Results and discussion}

\subsection{Chemical composition and microstructure}

The chemical composition of Ag-DLC coatings is shown in Table 1, together with the coatings' thicknesses, deposition rates and mechanical properties. The increase on the number of silver pellets placed into the erosion zone of graphite target resulted in an increase of silver content from 0 (for reference DLC coating) to 13.1 at.\%. The level of oxygen originating from residual atmosphere and target contamination was relatively low. To facilitate reading, the coatings were denominated based on their silver content (rounded), i.e. Ag6 is the coating with $\mathrm{Ag}$ content 6.1 at.\%.

The deposition rate of Ag-DLC layer increased with the number of Ag pellets from $0.50 \mu \mathrm{m} / \mathrm{h}$ for pure DLC coatings up to $0.80 \mu \mathrm{m} / \mathrm{h}$ for the highest Ag content (13.1 at.\%). This increase was due to higher sputtering yield of $\mathrm{Ag}$ (3.12 for Ar bombardment at $500 \mathrm{eV}$ ) compared to carbon (0.12 for Ar bombardment at $500 \mathrm{eV}$ ) [24]. In order to compare tribological results of the coatings, the total film thickness (considering the Ti/TiN/TiCN interlayer and DLC coating) was kept in the range of 1.4-1.7 $\mu \mathrm{m}$ (Table 1); the deposition time of Ag-DLC layer was gradually reduced from $200 \mathrm{~min}$ for pure DLC to $110 \mathrm{~min}$ for $\mathrm{Ag} 13$ coating.

The XRD patterns of Ag-DLC coatings are presented in Fig. 1. The main feature in the spectra was the peaks related to TiN (ICDD 01-087-0632), i.e. the adhesion improving interlayer. TiN was the only phase observed for all coatings except for Ag13, which showed a very broad peak at $45^{\circ}$ attributed to Ag (ICDD 01-087-0720). The

Table 1

Chemical composition, thickness, deposition rate and mechanical properties of Ag-DLC coatings.

\begin{tabular}{|c|c|c|c|c|c|c|c|c|c|}
\hline \multirow[t]{2}{*}{ Coating } & \multirow[t]{2}{*}{ Number of Ag pellets } & \multicolumn{3}{|c|}{$\begin{array}{l}\text { Chemical composition } \\
\text { (at.\%) }\end{array}$} & \multirow[t]{2}{*}{$\begin{array}{l}\text { Thickness } \\
(\mu \mathrm{m})\end{array}$} & \multirow[t]{2}{*}{$\begin{array}{l}\text { Deposition rate } \\
(\mu \mathrm{m} / \mathrm{h})\end{array}$} & \multirow[t]{2}{*}{$\begin{array}{l}\text { Hardness } \\
(\mathrm{GPa})\end{array}$} & \multirow[t]{2}{*}{$\begin{array}{l}\text { Residual stress } \\
(\mathrm{GPa})\end{array}$} & \multirow[t]{2}{*}{$\begin{array}{l}\text { Critical load, } \mathrm{C} 1 \\
(\mathrm{~N})\end{array}$} \\
\hline & & $\mathrm{Ag}$ & $C$ & 0 & & & & & \\
\hline DLC & 0 & 0 & 98.7 & 1.3 & 1.68 & 0.50 & 12.9 & 2.3 & 35 \\
\hline Ag1 & 1 & 1.3 & 96.6 & 2.1 & 1.54 & 0.51 & 13.0 & 2.4 & 42 \\
\hline $\mathrm{Ag} 4$ & 3 & 3.7 & 95.1 & 1.2 & 1.61 & 0.60 & 13.0 & & 45 \\
\hline Ag6 & 6 & 6.1 & 93.6 & 0.3 & 1.41 & 0.61 & 12.0 & 2.2 & 37 \\
\hline Ag13 & 11 & 13.1 & 86.1 & 0.8 & 1.41 & 0.80 & 9.3 & 1.4 & 41 \\
\hline
\end{tabular}


a
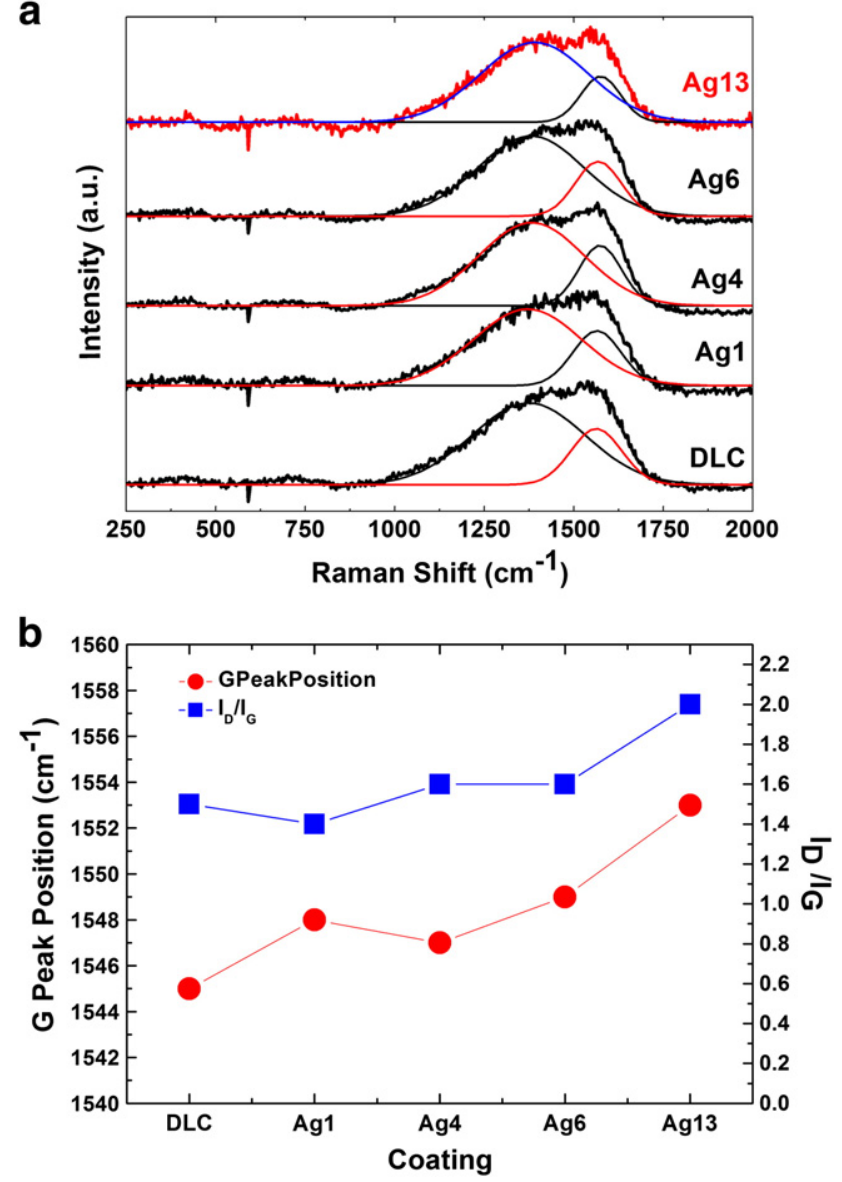

Fig. 2. a) Raman spectra of as-deposited Ag-DLC coatings, b) Variations of G peak position and $\mathrm{I}_{\mathrm{D}} / \mathrm{I}_{\mathrm{G}}$ ratio of $\mathrm{Ag}$-DLC coatings.

application of Scherrer's formula [25] to this peak estimated the silver grain size as 2-3 $\mathrm{nm}$. Obviously the absence of $\mathrm{Ag}$ peaks in the samples with Ag content below 13.1 at.\% cannot exclude the presence of small Ag clusters with sizes below $2 \mathrm{~nm}$, which are not detectable in XRD analysis. Silver carbides were neither expected nor observed and our results corroborate the studies on similar coating systems [15,26-28]. In fact, previous studies of DLC coatings alloyed with silver indicated that the microstructure of these coatings was characterized as random dispersion of metallic $\mathrm{Ag}$ nanoparticles in an amorphous C-matrix $[28,29]$. The size of these nanoparticles was related to the amount of $\mathrm{Ag}$ incorporated in the coatings.

Raman spectroscopy was performed in order to evaluate the effect of $\mathrm{Ag}$ incorporation on the carbon structure. Fig. 2a shows the Raman spectra of the coatings, where the two main peaks typical of carbon based coatings, located at around $1350 \mathrm{~cm}^{-1}$ (D peak) and $1550 \mathrm{~cm}^{-1}$ ( $G$ peak) could be identified [30]. In order to evaluate the variations in $\mathrm{I}_{\mathrm{D}} / \mathrm{I}_{\mathrm{G}}$ ratio and $\mathrm{G}$ peak position the spectra were fitted using two Gaussians and the $\mathrm{I}_{\mathrm{D}} / \mathrm{I}_{\mathrm{G}}$ ratio was evaluated taking into account the intensity ratio of $\mathrm{D}$ and $\mathrm{G}$ peaks [31]. The results presented in Fig. 2b show that the incorporation of Ag below 13.1 at.\% did not promote changes in the carbon atomic bond structure. On the other hand, the incorporation of 13.1 at.\% of Ag promoted an increase in the $I_{D} / I_{G}$ ratio and in the shift of $G$ peak position to higher wavenumber, which was attributed to an increase of graphite like bonds organized in rings $[28,30]$.

\subsection{Mechanical characterization}

Ag-DLC coatings' adhesion, hardness and residual stress are shown in Table 1 . The critical loads were determined according to the criteria presented by Zaidi et al. [32], where the first critical load, $\mathrm{C} 1$ represented the first visible damage (adhesive failure) in the coating and $\mathrm{CR}$ was the complete coating failure. All the tested coatings showed a CR value above $50 \mathrm{~N}$, while the $\mathrm{C} 1$ values were in the range 35-43 N. Although there is no clear dependence between the coating adhesion and the silver content, it is evident that the silver-containing coatings showed higher critical loads compared to pure DLC. The optical micrographs of the scratch tracks of DLC and
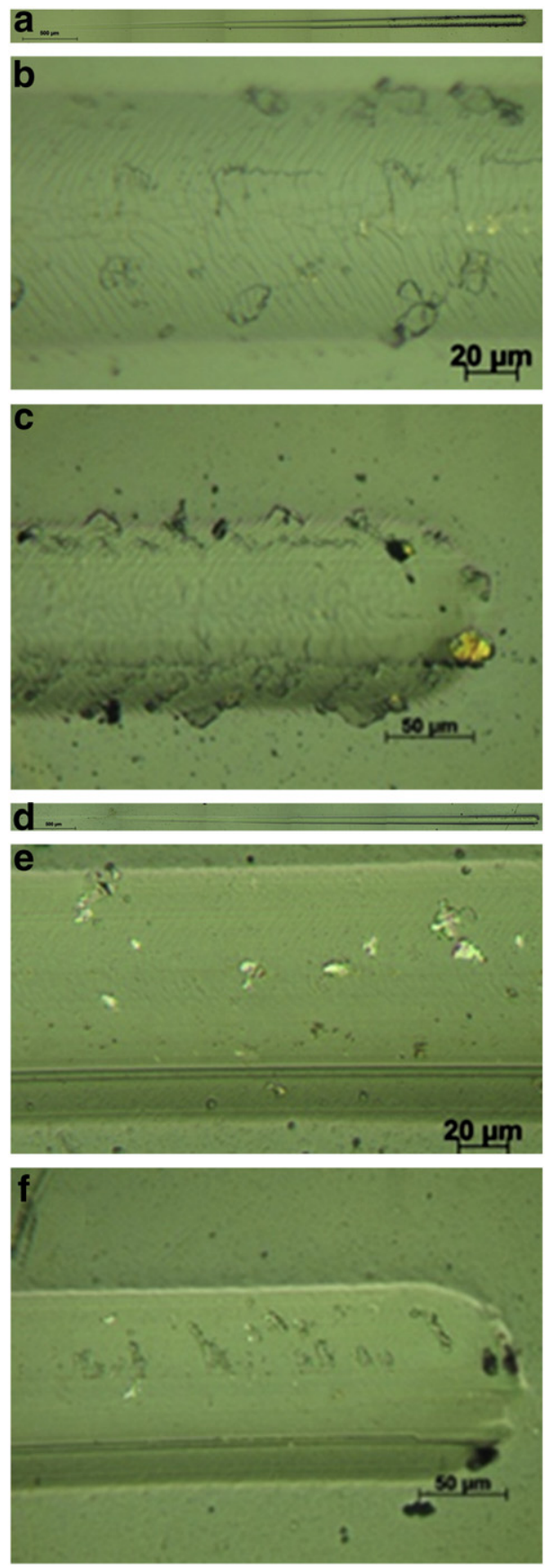

Fig. 3. Scratch tracks of sample a) DLC, b) detailed view of C1 (DLC), and c) detailed view of the end of the wear track (DLC). Scratch tracks of sample d) Ag13, e) detailed view of $\mathrm{C} 1$ (Ag13), and f) detailed view of the end of the wear track (Ag13). 


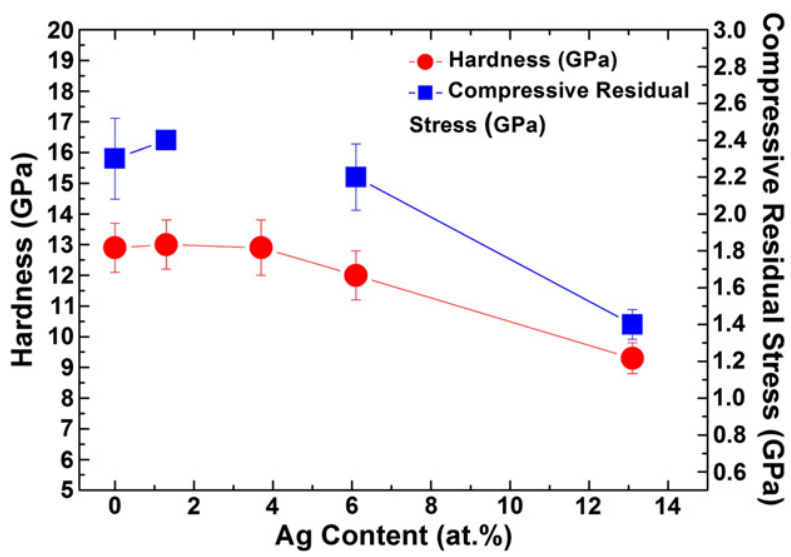

Fig. 4. Variations of Ag-DLC coatings' hardness and residual stress with increasing Ag content.

Ag13 coatings shown in Fig. 3, clearly demonstrate that the presence of buckling type failures disappeared in the case of Ag13 coating. The presence of a ductile phase $(\mathrm{Ag})$ in the carbon matrix could act as ductile bridges able to absorb the energy during the deformation process, which results in a lower density of detachment points and absence of buckling type failure.

The hardness and compressive residual stress as a function of $\mathrm{Ag}$ content follow a similar trend, see Fig. 4. The hardness and residual stress of pure DLC and low silver content coatings are almost identical with values 12-13 GPa and 2.2-2.4 GPa, respectively. Ag13 coating showed significantly lower values of hardness (9.3 GPa) and residual stress $(1.4 \mathrm{GPa})$. The drop in hardness for high silver content is well correlated with the changes in the coating structure. The hardness of carbon based coatings is mainly controlled by the structure and binding energy of carbon atoms, being accepted that a higher $\mathrm{sp}^{3} / \mathrm{sp}^{2}$ ratio results in higher hardness [1]. The Raman spectra, referred above showed that the incorporation of Ag below 13.1 at.\% did not promote any significant changes in the carbon matrix. Moreover, the formation of crystalline Ag phases was not detected for coatings with Ag contents below 13.1 at.\%, in other words, the size of silver nanoclusters (if they were present) was below $2 \mathrm{~nm}$, a size too small to significantly affect the coating's mechanical properties. Thus, larger soft Ag clusters observed in Ag13 coating combined with the formation of graphite-like bonds in the carbon matrix promote a decrease in the hardness.

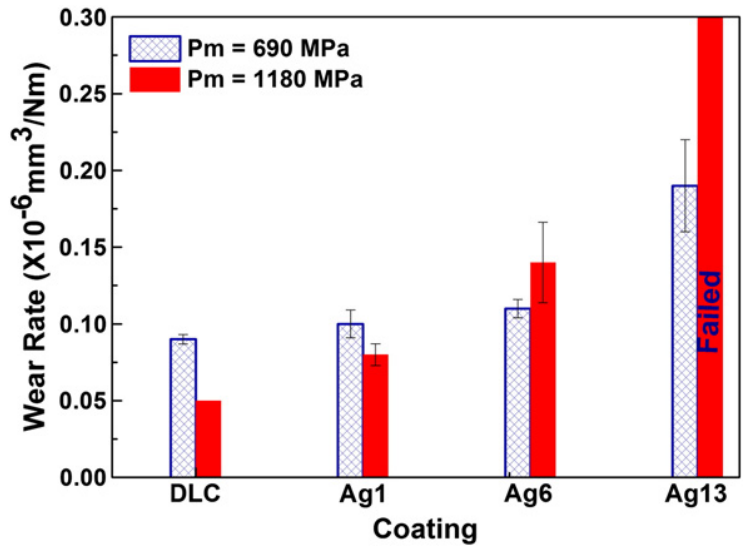

Fig. 5. Wear rate of the coatings with different Ag contents for Hertzian contact pressures of $\mathrm{Pm}=690 \mathrm{MPa}$ and $\mathrm{Pm}=1180 \mathrm{MPa}$.

\subsection{Tribological characterization}

The tribological properties (wear rate and friction coefficient) of the coatings were evaluated in dry sliding conditions with zirconia balls as counterparts. The values of the wear rate and friction curves are shown in Figs. 5 and 6, respectively. For lower contact pressure $(\mathrm{Pm}=690 \mathrm{MPa})$ the wear rate of DLC and low Ag content Ag-DLC coatings was similar (in the order of $0.10 \times 10^{-6} \mathrm{~mm}^{3} / \mathrm{Nm}$ ), although slightly higher in silver containing films, in good agreement with their similar structure and mechanical properties. For the coating with Ag content of 13.1 at.\% the wear rate almost doubled, showing a value of $0.19 \times 10^{-6} \mathrm{~mm}^{3} / \mathrm{Nm}$. Friction curves follow the same trend, i.e. low Ag content films show constant friction coefficients (CoFs), in a lower range from 0.10 to 0.14 (again values are slightly higher for Ag-containing films), whereas Ag13, although starting also at a low value, CoF increases significantly reaching in the end values above 0.2. At higher Hertzian contact pressure $(\mathrm{Pm}=$ $1180 \mathrm{MPa}$ ), different trends could be noticed: (i) the wear rate steadily increased with $\mathrm{Ag}$ content, showing $\mathrm{Ag} 13$ signs of complete destruction as suggested by the irregular and very high values achieved for CoF after, approximately, 12000 cycles; (ii) in relation to the $690 \mathrm{MPa}$ contact pressure, for low Ag content films, the wear rate has lower values, occurring the inverse for high $\mathrm{Ag}$ contents; (iii) excepting for $\mathrm{Ag} 13, \mathrm{CoF}$ values are also lower than that of $690 \mathrm{MPa}$ contact pressure, being the lowest for pure DLC coating.

The decrease in the wear rate and friction coefficient of DLC coatings in more severe test conditions, as it was observed for low $\mathrm{Ag}$ content films, has been reported by several authors [33,34]. In fact, the low friction coefficient of DLC coatings is attributed to the formation of a transfer layer on the counterpart; its formation is enhanced at higher contact pressures and sliding velocities [33]. The friction
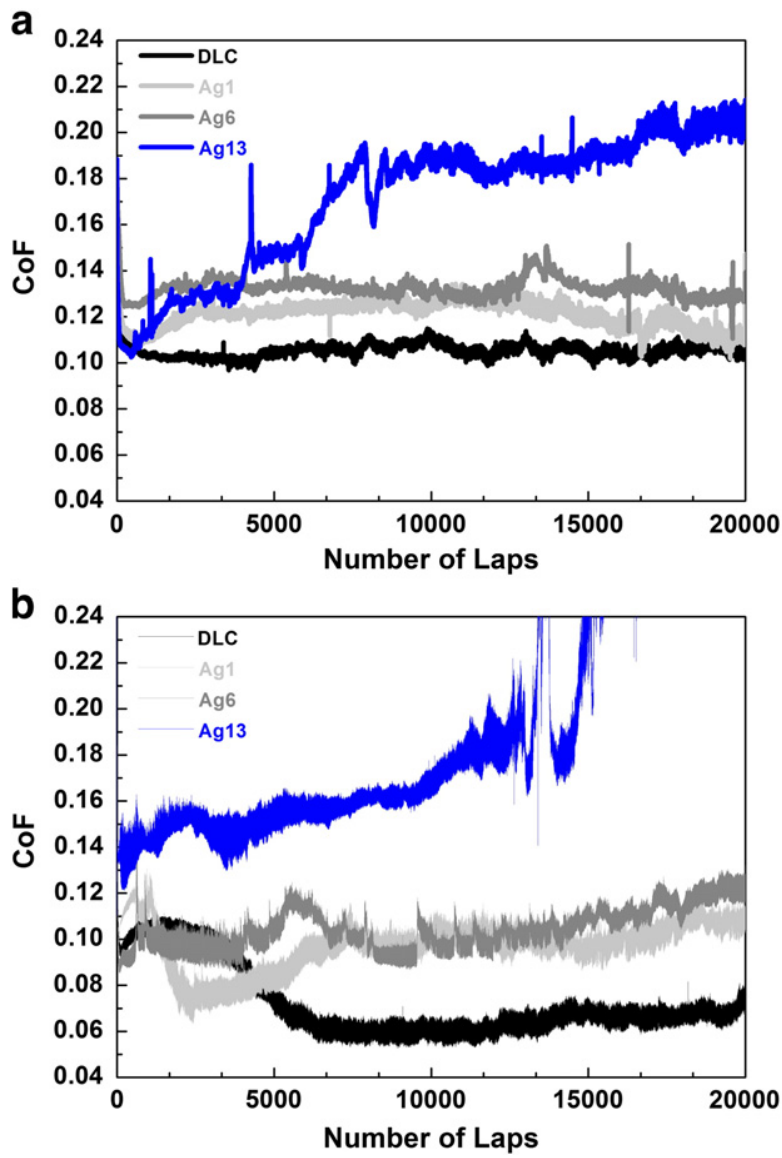

Fig. 6. $\mathrm{CoF}$ of Ag-DLC coatings tested at Hertzian contact pressures of a) $690 \mathrm{MPa}$ and b) $1180 \mathrm{MPa}$. 
a

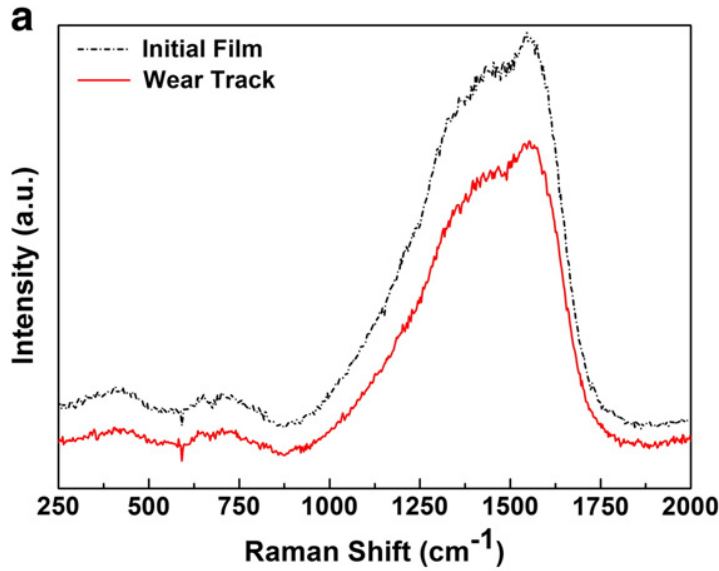

b

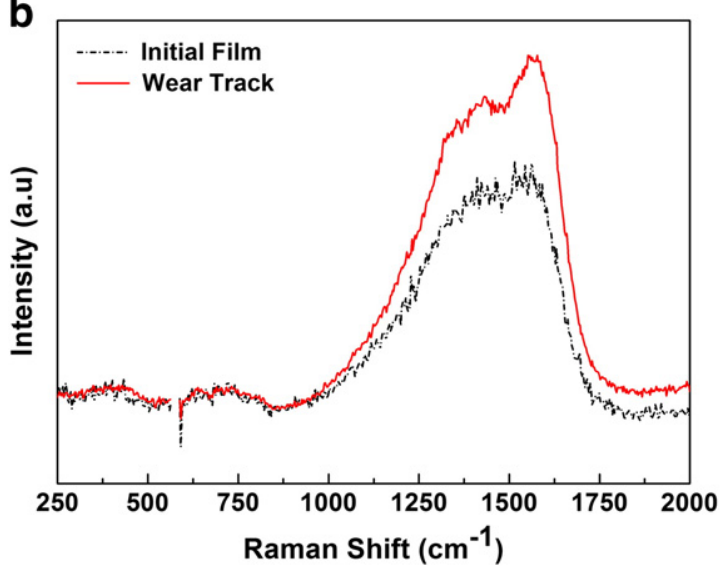

Fig. 7. Raman spectra of the wear track at Hertzian contact pressure of $\mathrm{Pm}=1180 \mathrm{MPa}$ of coatings a) DLC and b) Ag6.

coefficient of DLC at lower initial contact pressure ( $\mathrm{Pm}=690 \mathrm{MPa})$ reached a steady state regime after few laps and the value of $\mathrm{CoF}$ remained at 0.10 , whereas for the higher contact pressure the running in period was prolonged to approximately 5000 laps, where the friction coefficient stabilized close to 0.05 . This reduction in friction and wear corresponds to the formation of a compact transfer layer on the counterpart. In general, the friction coefficient of silver-containing coatings followed the same trend as DLC when the contact pressure was higher, (nevertheless the friction coefficient of Ag-DLC coatings was always higher than that of DLC). It is evident that high wear of $\mathrm{Ag} 13$ coating is related to higher friction; based on Fig. 6b we can speculate that the coating was worn through after approximately 12000 laps. The increase in the graphitic-like bond observed by Raman spectroscopy for the coating with silver content of 13 at.\% might suggest lower friction. However, the frictional mechanism completely changed when silver was added to DLC. The carbon-based tribolayer, which was formed during sliding of DLC coatings, was replaced by the Ag rich transfer layer formed on the ball counterface as demonstrated below in SEM analysis. Strong adhesion of the metallic transferred layer and the silver-rich areas in the coating wear track, as suggested by SEM analysis (Figs. 8 and 9), increased the friction.

In order to obtain information about the processes in sliding contact Raman analysis of DLC and Ag6 coatings was performed on the worn surface and outside the wear track as shown in Fig. 7a and b, respectively. The results did not indicate any significant changes in DLC structure during the sliding process. Counterparts sliding against Ag6 and Ag13 at $\mathrm{Pm}=690 \mathrm{MPa}$ were investigated together with corresponding wear tracks by SEM shortly after the tribological tests, see Figs. 8 and 9. The micrographs of the transfer layer on the counterparts sliding against the Ag6 (Fig. 8a) and Ag13 (Fig. 8b) coatings suggested relatively large

areas rich in silver, which should appear much brighter than carbon in BSE mode, due to the higher atomic mass of $\mathrm{Ag}$ in relation to carbon. The wear track of Ag6 coating shown in Fig. 9a is covered by Ag aggregates combined with smaller $\mathrm{Ag}$ clusters. The accumulation of $\mathrm{Ag}$ in the delamination zone was evident as well as the formation of the $\mathrm{Ag}$ particles on the wear track borders, possibly due to their detachment from the delamination zone during the sliding process. It should be pointed out that the delamination zone only presented local failure; however the clear observation of Ag accumulation in the wear track is clearly visible. SEM analysis of the worn surfaces performed again four months after the pin-on-disk test revealed that the amount of Ag on the coating surface was enhanced. In fact, the wear tracks of the Ag6 coating were totally covered by Ag, whereas other parts of the coating surface showed only small number of randomly distributed Ag particles (Fig. 9b). The micrograph in the inset in Fig. 9b was taken in the same zone in BSE mode, which allows stating that the bright aggregates found in the surface are related to Ag. Randomly oriented scratches on the surface were consequence of sample handling, which removed loosely adhered Ag particles. A similar behavior was found for coating Ag13, (see Fig. 9c), where the accumulation of $\mathrm{Ag}$ in the delamination zone (not representative of the wear behavior for Pm $=690 \mathrm{MPa}$ ) was observed, combined with silver aggregates at the wear track borders.

According to the results obtained from SEM analysis the formation of Ag rich transfer layer was observed as well as the accumulation of $\mathrm{Ag}$ in the wear track, which resulted in high CoF values when compared with the reference DLC coating. When incorporated into the matrix of hard coatings, such as TiCN [18], CrN [35], SiC and HfC [36], silver acts as a solid lubricant. However, hard nitride or carbide based coatings typically show much higher friction (typically $0.7-1$ ) than DLC. In the latter case the friction is much lower (approx. 0.1) due to the formation of a carbon tribolayer. When the carbon-based tribolayer is replaced by silver one, the friction inevitably increases.

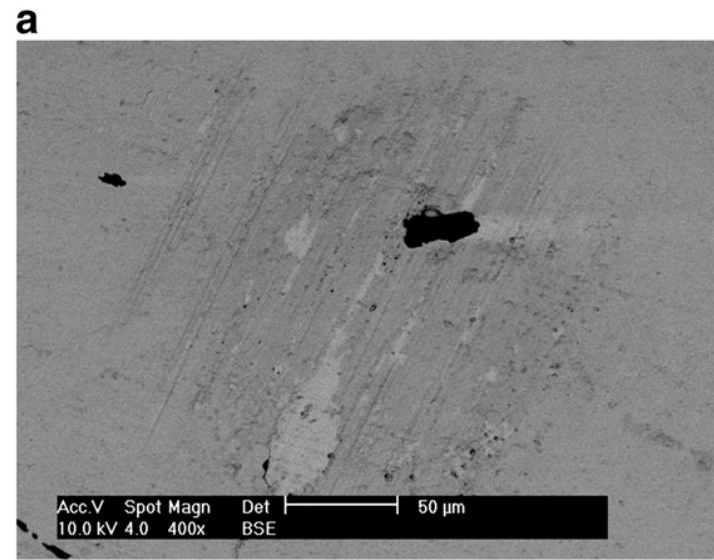

b

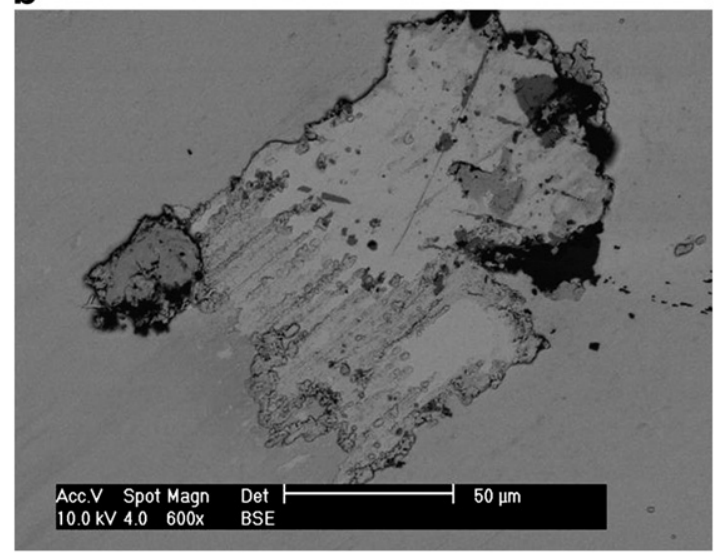

Fig. 8. SEM analysis of zirconia counterparts sliding against a) Ag6 and b) Ag13. 


\section{a}

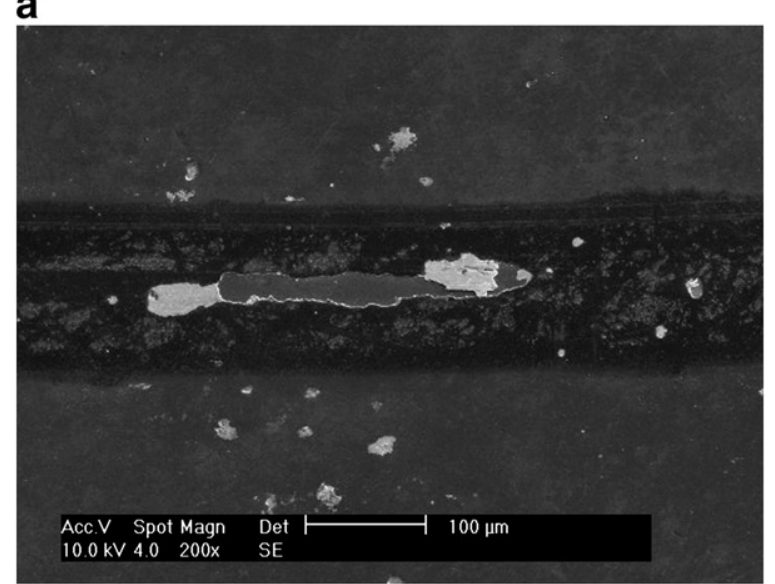

b

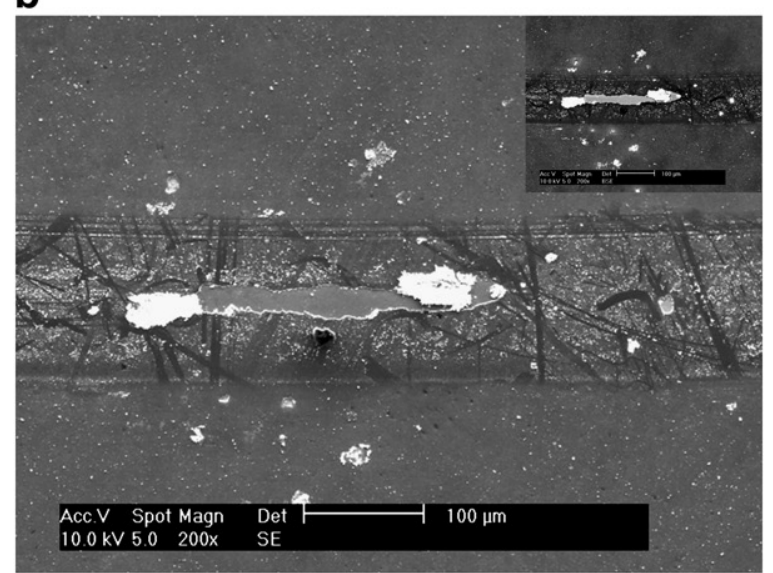

C

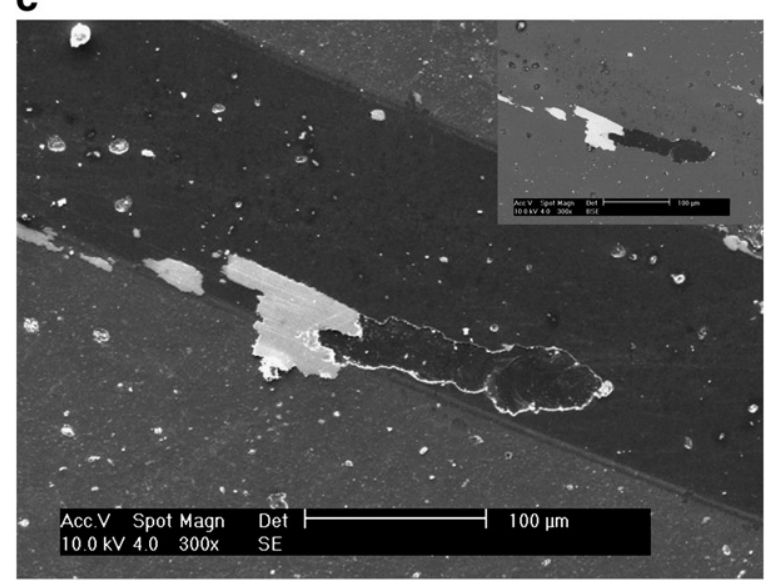

Fig. 9. SEM micrographs of the wear track of coating Ag6 a) observed shortly after the pin-on-disk test in SE mode and b) four months after pin-on-disk test in SE mode (inset taken in BSE mode) and c) wear track of coating Ag13 shortly after pin-on-disk test in SE mode (inset in BSE mode).

The original surface of coating Ag13 was observed by SEM analysis in order to determine the mechanisms of Ag transfer layer formation and silver accumulation on the wear track. The SEM images of the surface of coating Ag13 are presented in Fig. 10. SEM analysis was performed 6 months after coating deposition and it was found that the original gray color of the coatings changed to bright silver like appearance. From Fig. 10 it can be found that the coating's surface is covered by small particles present in the surface combined with randomly oriented scratches originated by the sample handling. The EDS analysis performed inside the scratched region (Z1) and on the

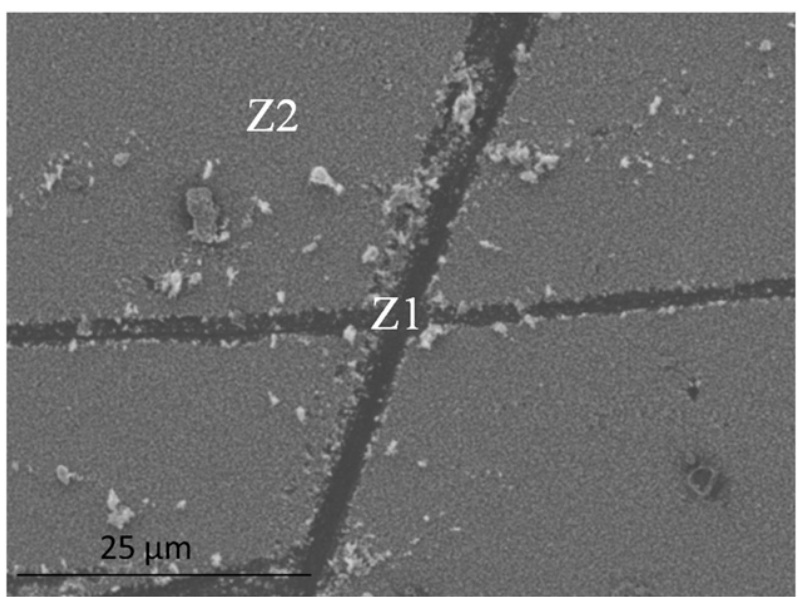

Fig. 10. SEM micrograph of coating Ag13 (analysis performed 6 months after coating deposition).

original surface (Z2) suggests that the surface particles are Ag. In fact an increase in silver content from 22 at.\% to 30 at.\% was found along with a decrease in carbon content from 78 at.\% to 70 at.\% (see Table 2). The presence of these soft aggregates on the surface leads to the formation of Ag transfer layer and accumulation on the wear track during the sliding process. The changes on the coating surface along the time, suggested by the enrichment of $\mathrm{Ag}$ inside the wear track (Fig. 9b) as well as the changes observed in sample Ag13 suggest that silver is segregating in the coating surface. The surface segregation of $\mathrm{Ag}$ has been reported by several authors; however this process occurred at higher temperatures [35,37-40]. The surface segregation of silver is attributed to the low miscibility of this noble metal in different coatings [37] associated with the reduction in the system surface energy, which drives the segregation process. In the case of DLC matrix it seems that surface segregation occurs even at room temperature, however a more detailed study of this topic is needed in order to better understand the stability of Ag-DLC system. According to the results obtained it is presumed that Ag aggregates on the coating surface along the time, due to the diffusion of silver atoms present on the surface combined with some segregation of Ag atoms from the first nanometers below the surface, as proposed in the scheme of Fig. 11. The variations on the worn surface along the time indicate that the $\mathrm{Ag}$ accumulates inside the wear track which may be associated to the diffusion of $\mathrm{Ag}$ from the layers below the surface, as proposed in the scheme of Fig. 11. From the SEM images depicted in Fig. 9b it can be found that the amount of silver inside the wear track is higher in relation to the as deposited coating. However, since the coatings' surface was cleaned before the pin-on-disk test the segregated particles were removed, which explains the lower amount of silver outside the wear track.

\section{Conclusion}

Ag-DLC coatings with silver contents ranging from 1.3 at.\% to 13.1 at.\% were deposited by DC magnetron sputtering. The Raman results revealed that the incorporation of $\mathrm{Ag}$ up to $6.1 \mathrm{at} . \%$ did not promote any changes in the carbon matrix and no $\mathrm{Ag}$ phases were detected by XRD analysis, which allows to conclude that silver

Table 2

Chemical composition of Z1 and Z2 determined by EDX analysis.

\begin{tabular}{lll}
\hline & Ag content (at.\%) & C content (at.\%) \\
\hline Z1 & 22 & 78 \\
Z2 & 30 & 70 \\
\hline
\end{tabular}


a
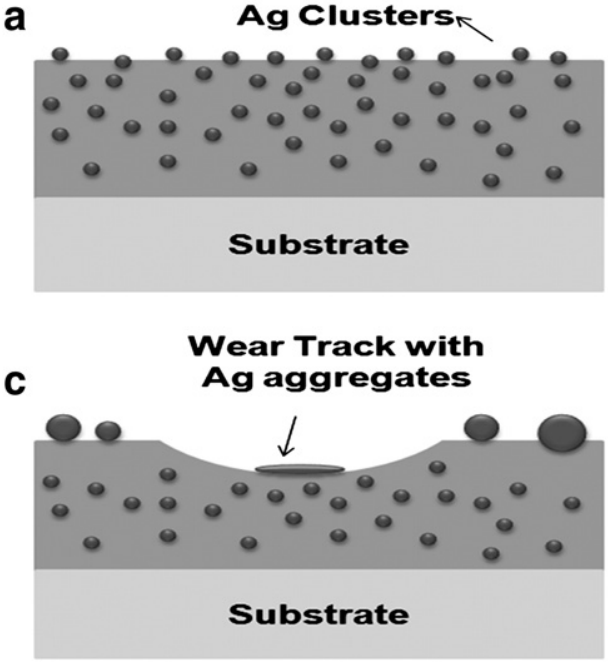

b

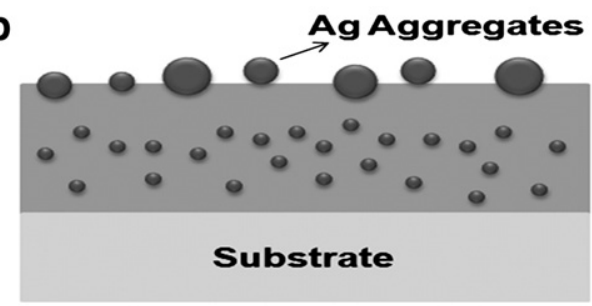

d

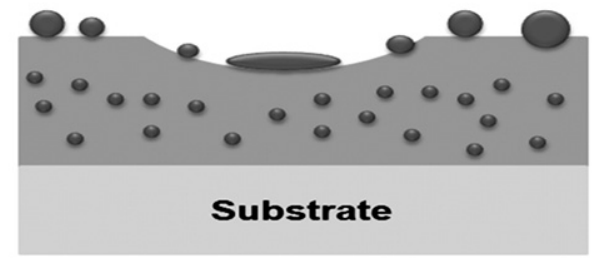

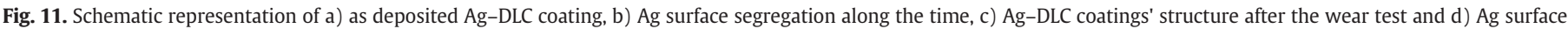
segregation inside the wear track along the time.

atoms are probably dispersed in the carbon matrix forming very small nanoclusters. The increase of $\mathrm{Ag}$ to 13.1 at.\% leads to the formation of crystalline Ag phase with a grain size in the order of $2-3 \mathrm{~nm}$, which resulted in an increase of graphite like bonds organized in rings. The variations in coating structures are well correlated with the changes in the mechanical properties, being observed that only the incorporation of 13.1 at.\% Ag promoted changes, a decrease on both compressive residual stress and hardness.

In order to evaluate the tribological behavior of these coatings, pin-on-disk tests were performed against a zirconia counterpart at two different contact pressures: $690 \mathrm{MPa}$ and $1180 \mathrm{MPa}$. For the lower contact pressure only a slight increase in the wear rate was observed with the incorporation of $\mathrm{Ag}$ up to $6.1 \mathrm{at} . \%$, while increasing the contact pressure to $1180 \mathrm{MPa}$ promoted a higher increase in the wear rate of $\mathrm{Ag}$ doped coatings, in relation to DLC coating. From SEM analysis it was concluded that Ag aggregates on the worn surface during the sliding process are forming Ag layer on the counterpart, which may be responsible for the increase in the friction coefficient and wear rate in relation to the reference DLC coating. SEM analysis of the coating surface performed along the time, suggests the segregation of Ag on the coating's surface, which seems to play a major role in the tribological behavior of Ag-DLC coatings, thus masking the intrinsic influence of the structural and chemical properties on the tribological behavior. In fact, the formation of $\mathrm{Ag}$ aggregates on the wear track, combined with the formation of $\mathrm{Ag}$ rich transfer layer on the counterpart are responsible for the higher friction coefficient and wear rate of Ag doped coatings at higher contact pressure, which formation might be enhanced by the Ag segregation process.

\section{Acknowledgments}

The work was partially funded by the Czech Science Foundation through the project $108 / 10 / 1782$ and by the national funds through FCT - Fundação para a Ciência e Tecnologia under the grant SFRH/ $\mathrm{BD} / 82472 / 2011$ and co-funded by the FSE. This research is sponsored by the FEDER funds through the program COMPETE - Programa Operacional Factores de Competitividade and by the national funds through FCT - Fundação para a Ciência e Tecnologia in the framework of the Strategic Projects PEST-C/EME/UIO0285/2011.

\section{References}

[1] C.A. Charitidis, Int. J. Refract. Met. Hard Mater. 28 (2010) 51-70.

[2] R.M. Balestra, A.M.G. Castro, M. Evaristo, A. Escudeiro, Petr Mutafov, T. Polcar, A. Cavaleiro, Surf. Coat. Technol. 205 (2011) S79-S83.
[3] J.C. Sanchez-Lopez, C. Donnet, J. Fontaine, M. Belin, A. Grill, V. Patel, C. Jahnes, Diamond Relat. Mater. 9 (2000) 638-642.

[4] V.M. Tiainen, Diamond Relat. Mater. 10 (2001) 153-160.

[5] J.I. Oñate, M. Comin, I. Braceras, A. Garcia, J.L. Viviente, M. Brizuela, N. Garagorri, J.L. Peris, J.I. Alava, Surf. Coat. Technol. 142-144 (2001) 1056-1062.

[6] K. Gutensohn, C. Beythien, J. Bau, T. Fenner, P. Grewe, R. Koester, K. Padmanaban, P. Kuehnl, Thromb. Res. 99 (2000) 577-585.

[7] L.J. Yu, X. Wang, X.H. Wang, X.H. Liu, Surf. Coat. Technol. 128-129 (2000) 484-488.

[8] W. Jacob, W. Möller, Appl. Phys. Lett. 63 (1993) 1771-1773.

[9] J. Musil, M. Louda, Z. Soukup, M. Kubásek, Diamond Relat. Mater. 17 (2008) 1905-1911.

[10] J.C. Sánchez-López, A. Fernández, in: C. Donnet, A. Erdemir (Eds.), Tribology of Diamond-like Carbon Films, Springer, New York, 2008, pp. 311-338.

[11] A. Escudeiro, T. Polcar, A. Cavaleiro, Vacuum 85 (2011) 1144-1148.

[12] C.W. Moura e Silva, J.R.T. Branco, A. Cavaleiro, Thin Solid Films 515 (2006) 1063-1068.

[13] W. Dai, G. Wu, A. Wang, Diamond Relat. Mater. 19 (2010) 1307-1315.

[14] A. Escudeiro, et al., Thin Solid Films (2013), http://dx.doi.org/10.1016/j.tsf.2012.12.086

[15] X. Yu, Y. Qin, C.B. Wang, Y.Q. Yang, X.C. Ma, Vacuum 89 (2013) 82-85.

[16] Y. Xiang, W. Cheng-biao, P. Zhi-jian, Key Eng. Mater. 434-435 (2010) 462-465.

[17] H.W. Choi, J.H. Choi, K.R. Lee, J.P. Ahn, K.H. Oh, Thin Solid Films 516 (2007) 248-251.

[18] J.C. Sánchez-López, M.D. Abad, I. Carvalho, R. Escobar Galindo, N. Benito, S. Ribeiro, M. Henriques, A. Cavaleiro, S. Carvalho, Surf Coat. Technol. 206 (2012) 2192-2198.

[19] P.J. Kelly, H. Li, P.S. Benson, K.A. Whitehead, J. Verran, R.D. Arnell, I. Iordanova, Surf. Coat. Technol. 205 (2010) 1606-1610.

[20] J.H. Hsieh, C.C. Tseng, Y.K. Chang, S.Y. Chang, W. Wu, Surf. Coat. Technol. 202 (2008) 5586-5589.

[21] M.L. Morrison, R.A. Buchanan, P.K. Liaw, C.J. Berry, R.L. Brigmon, L. Riester, H Abernathy, C. Jin, R.J. Narayan, Diamond Relat. Mater. 15 (2006) 138-146.

[22] G.G. Stoney, Proc. R. Soc. A 82 (1909) 172

[23] ASTM G99-05, Standard Test Method for Wear Testing with a Pin-on-Disk Apparatus, 2010.

[24] J.L. Vossen, J.J. Cuomo, in: J.L. Vossen, W. Kern (Eds.), Thin Film Processes, Academic Press, 1978, pp. 12-73.

[25] P. Scherrer, N.G.W. Gottingen, Math.-Phys. Kl. 2 (1918) 96.

[26] N.K. Manninen, R. Escobar Galindo, N. Benito, N.M. Figueiredo, A. Cavaleiro, S. Carvalho, J. Phys. D: Appl. Phys. 44 (2011) 375501.

[27] H.-S. Zhang, J.L. Endrino, A. Anders, Appl. Surf. Sci. 255 (2008) 2551-2556.

[28] H.W. Choi, R.H. Dauskardt, S.-Cheol Lee, K.-Ryeol Lee, K.H. Oh, Diamond Relat. Mater. 17 (2008) 252-257.

[29] Y. Wang, J. Wang, G. Zhang, L. Wang, P. Yan, Surf. Coat. Technol. 206 (2012) 3299-3308.

[30] J. Roberston, Mater. Sci. Eng. R 37 (2002) 129-281.

[31] A.C. Ferrari, J. Robertson, Phys. Rev. B 61 (20) (2000) 14095.

[32] H. Zaidi, A. Djamai, K.J. Chin, T. Mathia, Tribol. Int. 39 (2006) 124-128.

[33] H. Ronkainen, K. Holmberg, in: C. Donnet, A. Erdemir (Eds.), Tribology of Diamond-like Carbon Films, Springer, New York, 2008, p. 170.

[34] D. Camino, A.H.S. Jones, D. Mercs, D.G. Teer, Vacuum 52 (1999) 125-131.

[35] L. Incerti, A. Rotaa, S. Valeri, A. Miguel, J.A. García, R.J. Rodríguez, J. Osés, Vacuum 85 (2011) 1108-1113.

[36] J.E. Krzanowski, J.L. Endrino, J.J. Nainaparampil, J.S. Zabinski, J. Mater. Eng. Perform. 13 (4) (2004) 439-444.

[37] J.J. Hu, C. Muratore, A.A. Voevodin, Compos. Sci. Technol. 67 (2007) 336-347.

[38] F. Niu, I.T.H. Chang, P.J. Dobson, B. Cantor, Mater. Sci. Eng., A 226-228 (1997) 161-167.

[39] C.P. Mulligan, D. Gall, Surf. Coat. Technol. 200 (2005) 1495-1500.

[40] C.W. Bates Jr., Q.Y. Chen, Mater. Lett. 23 (1995) 7-12. 